\title{
Feature selection for portfolio optimization
}

\section{Bjerring, Thomas Trier; Ross, Omri; Weissensteiner, Alex}

\section{Published in:}

Annals of Operations Research

Link to article, DOI:

$10.1007 / \mathrm{s} 10479-016-2155-y$

Publication date:

2017

Document Version

Peer reviewed version

Link back to DTU Orbit

\section{Citation (APA):}

Bjerring, T. T., Ross, O., \& Weissensteiner, A. (2017). Feature selection for portfolio optimization. Annals of Operations Research, 256(1), 21-40. https://doi.org/10.1007/s10479-016-2155-y

\section{General rights}

Copyright and moral rights for the publications made accessible in the public portal are retained by the authors and/or other copyright owners and it is a condition of accessing publications that users recognise and abide by the legal requirements associated with these rights.

- Users may download and print one copy of any publication from the public portal for the purpose of private study or research.

- You may not further distribute the material or use it for any profit-making activity or commercial gain

- You may freely distribute the URL identifying the publication in the public portal

If you believe that this document breaches copyright please contact us providing details, and we will remove access to the work immediately and investigate your claim. 


\title{
Feature Selection for Portfolio Optimization
}

\author{
Thomas Trier Bjerring \\ Technical University of Denmark Technical University of Denmark and \\ University of Copenhagen \\ Alex Weissensteiner \\ Technical University of Denmark and \\ Free University of Bozen-Bolzano
}

January 26, 2016

\begin{abstract}
Most portfolio selection rules based on the sample mean and covariance matrix perform poorly out-of-sample. Moreover, there is a growing body of evidence that such optimization rules are not able to beat simple rules of thumb, such as $1 / \mathrm{N}$. Parameter uncertainty has been identified as one major reason for these findings. A strand of literature addresses this problem by improving the parameter estimation and/or by relying on more robust portfolio selection methods. Independent of the chosen portfolio selection rule, we propose using feature selection first in order to reduce the asset menu. While most of the diversification benefits are preserved, the parameter estimation problem is alleviated. We conduct out-of-sample back-tests to show that in most cases different well-established portfolio selection rules applied on the reduced asset universe are able to improve alpha relative to different prominent factor models.
\end{abstract}

Keywords: Portfolio Optimization, Parameter Uncertainty, Feature Selection, Agglomerative Hierarchical Clustering

\section{Introduction}

The seminal work of Markowitz (1952) has inspired a lot of work in the field of asset allocation. However, the solutions obtained by such techniques are usually very sensitive to the input parameters (see e.g. Best and Grauer, 1991) with the consequence that estimation errors lead to unstable and extreme positions in single assets. Chopra and Ziemba (1993) are one of the first to quantify the consequences of misspecified parameters in asset allocation decisions. Specifically, they illustrate that in their setting errors 
in expected returns are about ten times more important than errors in variances and covariances. Furthermore, in addition to the general consensus that expected returns are notoriously difficult to predict, Merton (1980) shows that even if the true parameters were constant, very long time series would be required to estimate expected returns in a reliable way. As a consequence, a trading strategy based on the sample minimum variance portfolio, which completely abstains from estimating expected returns, shows a better risk-adjusted performance than many other portfolio selection rules (see e.g. Haugen and Baker, 1991; Clarke et al., 2006; Scherer, 2011). Others propose different techniques to alleviate the problem of estimating expected returns. Jorion (1986) considers explicitly the potential utility loss when using sample means to estimate expected returns. In order to minimize this loss function, he uses Bayes-Stein estimation to shrink the sample means toward a common value. A simulation study shows that this correction provides significant gains. Black and Litterman (1992) argue that the only sensible "neutral" expected returns are those that would clear the market if all investors had identical views. Hence, the natural choice are the equilibrium expected returns derived from reverse optimization using the current market capitalization. Having these "neutral expected returns" as a starting point, they illustrate how to combine them with an investor's own view in a statistically consistent way.

Kan and Zhou (2007) show that there is a very significant interactive effect between the estimation of the parameters and the ratio of the number of assets to the number of observations. If the number of assets is small compared to the number of observations, then the estimation of expected returns is more important (in line with Chopra and Ziemba, 1993). However, when this fraction grows, then estimation errors in the sample covariance grow too, and may become more severe in terms of utility costs than the estimation errors in expected returns. Furthermore, when the number of assets exceeds the number of observations, the sample covariance matrix is always singular (even if the true covariance matrix is known to be non-singular). Many papers address the problem of estimating the covariance matrix from limited sample data.

Ledoit and Wolf $(2003,2004 a, b, 2012)$ propose using the "shrinkage" technique in order to pull extreme coefficients in the sample covariance matrix, which tend to contain a lot of error, towards more central values of a highly structured estimator. They derive the optimal shrinkage intensity in terms of a loss function, and they suggest using factor models ${ }^{1}$ or constant correlation models as structured estimators. Given that weight constraints improve the performance of mean-variance efficient portfolios, Jagannathan and Ma (2003) study the short-sale constrained minimum-variance port-

\footnotetext{
${ }^{1}$ See Kritzman (1993) who compares factor analysis and cross-sectional regression for that purpose.
} 
folio. They show that the optimal solution under short-sale constraints corresponds to the optimal solution of the unconstrained problem if shrinkage is used to estimate the covariance matrix, i.e. there is a one-to-one relationship between short-sale constraints and the shrinkage technique. DeMiguel et al. (2009a) generalize these results by solving the classical minimum-variance problem under norm-constrained asset weights. They show that their setting nests the shrinkage technique of Ledoit and Wolf (2003, 2004a) and Jagannathan and Ma (2003) as special cases. Given that for more volatile stocks the parameter estimation risk is higher, Levy and Levy (2014) propose two variance-based constraints to alleviate the problem of parameter uncertainty. First, the Variance-Based Constraints on the single weights, which are inversely proportional to the sample standard deviation of each asset. Second, the Global Variance-Based Constraints, where instead of sharp boundary constraints on each stock a quadratic "cost" is assigned to deviations from an equally weighted portfolio. Comparing ten optimization methods, they find that the two new suggested methods typically yield the best performance in terms of Sharpe ratio.

Another method to mitigate the estimation problem uses more portfolios than those proposed by the classical two-funds Tobin separation theorem. Kan and Zhou (2007) suggest adding a third risky fund to the risk-free asset and to the sample tangency portfolio to hedge against parameter uncertainty. In particular, under the assumption of constant parameters, they show that a portfolio which optimally combines the risk-free asset, the sample tangency portfolio (TP) and the sample global minimum-variance portfolio (MVP) dominates a portfolio with just the risk-free asset and the sample tangency portfolio.

The most extreme approach to address the problem of parameter uncertainty is to ignore all historical observations and to invest equally in the available assets. Such a strategy is known as the $1 / \mathrm{N}$ rule. Duchin and Levy (2009) use the 30 Fama-French industry portfolios (2001-2007) to compare the $1 / \mathrm{N}$ rule against a Markowitz meanvariance rule under short-sale constraints. They illustrate that for a low number of assets (below 25) the $1 / \mathrm{N}$ rule provides a higher average out-of-sample return. Only if all 30 assets are traded, then the classical optimization approach outperforms the $1 / \mathrm{N}$ rule slightly. DeMiguel et al. (2009b) compare 14 portfolio selection rules across seven empirical datasets and show that none is consistently better out-of-sample than the $1 / \mathrm{N}$ rule. Furthermore, under the assumption of constant parameters, they show that timeseries of extreme length (more than 6000 months for 50 assets) are necessary to beat the $1 / \mathrm{N}$ benchmark.

Given the results of DeMiguel et al. (2009b), Tu and Zhou (2011) combine the 1/N 
rule with four other well-known portfolio selection rules. Among others, they extend the Kan and Zhou (2007) model and propose adding the equally weighted 1/N portfolio as a fourth fund in an optimal way to reduce the estimation error. The MVP and the $1 / \mathrm{N}$ portfolio are natural candidates: While the MVP does not depend on expected returns, for the $1 / \mathrm{N}$ portfolio neither expected returns nor a covariance matrix have to be estimated. ${ }^{2}$

The results given by DeMiguel et al. (2009b) raise serious concerns about portfolio optimization altogether. In defense of optimization, Kritzman et al. (2010) argue that most studies rely on too short samples for estimating expected returns, which often yields implausible results. They show that when estimations of expected excess returns are based on long-term samples, then usually optimized portfolios outperform equally weighted portfolios.

To sum up: Many of the aforementioned papers illustrate that the problem of parameter uncertainty increases with the number of assets (see e.g. Kan and Zhou, 2007). Different data mining techniques such as factor models (see e.g. Kritzman, 1993), shrinkage of the mean (see e.g. Jorion, 1986) and shrinkage of the covariance (see e.g. Ledoit and Wolf, 2003, 2004a) are proposed to alleviate the problem of the parameter estimation. Under the assumption of constant parameters, extending the observation period improves the performance of optimization based portfolio rules (see e.g. DeMiguel et al., 2009b; Kritzman et al., 2010). However, whether parameters are really constant over time is questionable, which suggests that simply expanding the observation period might not be the best strategy in practice.

Compared to the above mentioned literature, in this paper we propose using feature selection by agglomerative hierarchical clustering. Based on correlation, we create groups of assets such that the similarity within a cluster and the dissimilarity between different clusters is maximized. From each group we select then one representative asset to construct a smaller but yet comprehensive enough universe. ${ }^{3}$ As the representative asset we use the medoid, whose average dissimilarity to all the objects in the cluster is minimal. While the reduced asset menu facilitates the estimation of the parameters, the chosen assets still allow to benefit from diversification. Our choice is motivated by previous studies. Tola et al. (2008) show that clustering algorithms can improve the reliability of the portfolio in terms of the ratio between predicted and realized risk. Lisi and Corazza (2008) use clustering for a practical portfolio optimization task under car-

\footnotetext{
${ }^{2}$ For the rest of the paper, when referring to the Tu and Zhou (2011) strategy, we mean the optimal combination of $1 / \mathrm{N}$ with Kan and Zhou (2007).

${ }^{3}$ In the following we use the term "feature selection" as synonym for "hierarchical clustering".
} 
dinality constraints. They use different distance functions and illustrate that in general clustering improves the out-of-sample performance compared to a benchmark. Nanda et al. (2010) compare different clustering techniques (as $K$-means, Fuzzy $C$-means, Self Organizing Maps) for portfolio management in the Indian market and report benefits compared to the benchmark (the Sensex index).

The present work makes four contributions to the literature on clustering in portfolio optimization. First, compared to the above mentioned papers, our out-of-sample back-tests are based on long and well-known time series. Specifically, we use the valueweighted 49 industry portfolios provided by Kenneth French ${ }^{4}$ as well as the constituent stocks of the S\&P 500. For both data sets, we use monthly returns from 1970 to 2013. Second, in addition to the classical minimum-variance portfolio and the tangency portfolio, we consider also the more advanced portfolio selection rules suggested by Kan and Zhou (2007) and Tu and Zhou (2011). We compare pairwise the results with and without feature selection. ${ }^{5}$ Furthermore, feature selection allows to use these portfolio selection rules also on data sets with more assets than observations. Third, in line with Kritzman et al. (2010) we highlight the importance of the length of the observation period by presenting back-test results for rolling windows of 5, 10, 15 and 20 years. Finally, we base our assessment on the alpha relative to the most prominent factor models, such as Fama and French (1993) and Carhart (1997). As a main result, we show that for most test cases the performance of the reduced asset universe improves. In particular, we show that the alpha of the $1 / \mathrm{N}$ strategy also benefits from reducing the asset menu. As the $1 / \mathrm{N}$ rule is not prone to parameter estimation errors, this result might be counterintuitive. We explain this finding with other beneficial properties of feature selection. First, in addition to alleviating the problems due to parameter estimation, the concentration risk of a portfolio is also reduced. To illustrate this point, consider the fact that over $20 \%$ of the stocks in the S\&P 500 are from the technology sector. As a result, the $1 / \mathrm{N}$ portfolio has high concentration risk in this sector. Feature selection forms groups such that the intra-group similarity and the inter-group dissimilarity is maximized, i.e. similar stocks are allocated to the same group. By choosing then a representative asset out of each group such sector-concentration risks are mitigated. Second, we show that for appropriate observation periods feature selection reduces the beta of the $1 / \mathrm{N}$ portfolio, which relates to the "betting-against-beta" idea proposed by Frazzini and Pedersen (2014).

\footnotetext{
${ }^{4}$ see http://mba.tuck.dartmouth.edu/pages/faculty/ken.french/data_library.html

${ }^{5}$ Given the focus of this paper, we want to point out that the proposed investigation is not intended as a horse race between the different portfolio selection rules.
} 
The paper is structured as follows. Section 2 summarizes the classical portfolio optimization techniques which are used in this paper. Section 3 offers a more detailed explanation of how we use feature selection for the problem at hand. Section 4 describes the data and the results of using feature selection in practice, and Section 5 concludes.

\section{Classical Mean-Variance Optimization}

Portfolio selection according to Markowitz is based on the assumption of multivariate normal asset returns. An investor, faced with the decision on how to allocate funds to $N$ risky and one riskless asset, optimizes the trade-off between the expectation and the variance of the portfolio returns. This preference can be formulated as

$$
\max _{w} w^{\top} \mu-\frac{\lambda}{2} w^{\top} \Sigma w
$$

where $w=\left(w_{1}, \ldots, w_{i}, \ldots, w_{N}\right)^{\top}$ represents the weights allocated to each risky asset in the portfolio, $\mu$ is the vector of expected excess returns over the risk free rate, $\lambda$ denotes the risk aversion coefficient, and $\Sigma$ is the variance-covariance matrix. Consequently, the difference $1-\mathbf{1}^{\top} w$ is invested in the riskless asset.

In practice the parameters $\mu$ and $\Sigma$ are unknown, i.e. the portfolio optimization has to be conducted under parameter uncertainty. Estimation errors can have a substantial influence on the out-of-sample performance of the model, and may lead to solutions that are far away from the true optimal portfolios (see DeMiguel and Nogales, 2009). In order to alleviate parameter uncertainty, Kan and Zhou (2007) propose a three-fund rule, which, in addition to the risk-free asset and the tangent portfolio, engages a third risky portfolio to hedge against the estimation risk. Furthermore, Tu and Zhou (2011) extend the three-fund rule and introduce the $1 / \mathrm{N}$ portfolio as a fourth portfolio.

DeMiguel et al. (2009b) show with a simulation study that the impact of parameter uncertainty on the performance of optimized portfolios depends heavily on the number of included assets. Given constant parameters, they illustrate that very long time series are needed to estimate $\mu$ and $\Sigma$ precisely enough to outperform an equally weighted portfolio. In reality, however, parameter values may vary over time, i.e. simply expanding the estimation window might induce the risk of using outdated observations.

Therefore, instead of simply expanding the window for the parameter estimation, in this paper we suggest a preliminary screening of the assets considered for optimization to reduce the dimensionality of the parameter estimation problem, and hereby improve the out-of-sample quality of the results. 
In order to assess the performance of feature selection, we compare pairwise the results of five different portfolio rules with and without reduction of the asset universe. More specifically, these asset allocation rules are the global minimum variance portfolio, the tangency mean-variance portfolio, the three-fund portfolio, the four-fund portfolio, and the $1 / \mathrm{N}$ portfolio. A short presentation of them is provided below.

\section{Global Minimum Variance Portfolio}

The minimum variance portfolio is a special case in the mean-variance portfolio framework, where the combination of risky assets is chosen such that the total variance of the portfolio returns is minimized, that is

$$
\begin{aligned}
& \min _{w} w^{\top} \Sigma w \\
& \text { s.t. } \mathbf{1}^{\top} w=1 .
\end{aligned}
$$

As this rule relies only on the estimation of the covariance matrix of asset returns, and ignores the expected returns, it is less prone to estimation errors as fewer parameters have to be estimated. Analytically, the weights of the minimum variance portfolio can be expressed as

$$
w_{M V}^{*}=\frac{\Sigma^{-1} \mathbf{1}}{\mathbf{1}^{\top} \Sigma^{-1} \mathbf{1}} .
$$

\section{Mean-Variance Tangency Portfolio}

Tobin (1958) expands Markowitz' seminal work for a risk-free asset and shows that the asset allocation task results in maximizing the Sharpe ratio of the portfolio

$$
\begin{aligned}
& \max _{w} \frac{w^{\top} \mu}{\sqrt{w^{\top} \Sigma w}} \\
& \text { s.t. } \mathbf{1}^{\top} w=1 .
\end{aligned}
$$

Thereafter, dependent on the investor's risk aversion, a combination between the resulting tangency portfolio and the risk-free asset is chosen. Analytically, the weights of the tangency portfolio are given by

$$
w_{T P}^{*}=\frac{\Sigma^{-1} \mu}{1^{\top} \Sigma^{-1} \mu} .
$$




\section{The Three-Fund Rule}

If the true mean and covariance of asset returns could be estimated precisely, as assumed in theory, then the two-fund separation would hold perfectly. However, when the parameters are unknown, the tangency portfolio is obtained with estimation errors. Intuitively, by using an additional risky portfolio the estimation problem can be alleviated. Kan and Zhou (2007) propose using the global minimum-variance portfolio as a third fund. As the estimation errors of the minimum variance portfolio and the tangency portfolio are not perfectly correlated, an optimal combination of them allows to improve the out-of-sample performance. The non-normalized weights of the combined portfolios are given by

$$
w_{K Z}=\frac{c_{3}}{\gamma}\left(c \Sigma^{-1} \mu+f \Sigma^{-1} \mathbf{1}\right),
$$

where $c$ and $f$ are chosen optimally to maximize the expected utility of a mean-variance investor given the relative risk aversion parameter $\gamma$ and the constant scalar $c_{3}=$ $\frac{(T-N-1)(T-N-4)}{T(T-2)}$. The allocation of funds to each of the risky portfolios depends on the number of assets $N$ and the length of the estimation window $T$. The more severe the parameter estimation problem, the higher the optimal proportion invested in the global minimum variance portfolio. In line with DeMiguel et al. (2009b), we set $\gamma$ equal to 1 , and we only focus on the composition of the risky part of the suggested portfolios. More specifically, we calculate the relative weights of the risky assets by

$$
w_{K Z}^{*}=\frac{w_{K Z}}{\left|\mathbf{1}^{\top} w_{K Z}\right|}
$$

where $\left|\mathbf{1}^{\top} w_{K Z}\right|$ guarantees that the direction of the portfolio position is preserved in cases where the sum of the weights of the risky assets is negative.

\section{The Four-Fund Rule}

DeMiguel et al. (2009b) show that the $1 / \mathrm{N}$ portfolio rule is difficult to outperform, especially if the observation period is short. However, as the $1 / \mathrm{N}$ rule makes no use of the sample information, it will fail to converge to the true optimal portfolio (unless, by chance, the two are the same). Therefore, if $1 / \mathrm{N}$ is far from the optimal portfolio its performance might be poor. Tu and Zhou (2011) propose the four-fund rule by combining the three fund-rule (see Kan and Zhou, 2007) in an optimal way with the $1 / \mathrm{N}$ portfolio. The non-normalized weights of this portfolio combination rule are

$$
w_{T Z}=(1-\delta) w_{e}+\delta w_{K Z}
$$


where $w_{e}$ is the equally weighted $(1 / \mathrm{N})$ portfolio and $w_{K Z}$ is the (non-normalized) optimal portfolio defined by the three-fund rule. The parameter $\delta$, which defines the ratio of wealth allocated to each of the portfolios, is determined by the number of assets $N$ and the number of observations $T$. The larger the number of assets relative to the number of observations, the more is invested in the $1 / \mathrm{N}$ portfolio, and the suggested portfolio becomes less prone to estimation errors. As in equation (4), we normalize the portfolio weights as

$$
w_{T Z}^{*}=\frac{w_{T Z}}{\left|\mathbf{1}^{\top} w_{T Z}\right|} .
$$

In general, the above mentioned papers assume unknown but constant parameters, which have to be estimated from historical observations. To mitigate the estimation problem, an increasing number of assets $N$ requires more observations $T$. In the limit - due to the Law of Large Numbers - the estimated parameters converge towards their true values. However, in case of time-varying investment opportunities and/or structural breaks, historical data may not correctly reflect the current state of the markets. On the other hand, for $N>T$, the sample covariance matrix is always singular. Therefore, it is natural to investigate whether it is beneficial to reduce the size of the asset menu and apply the portfolio rules to a representative subset, which reflects the overall dependence structure. The next section provides a detailed explanation (and a few examples) on how to reduce the asset universe using feature selection.

\section{Dimensionality Reduction Using Feature Selection}

This part of the paper proposes a heuristic, namely agglomerative hierarchical clustering, which exploits the underlying correlation structure of the complete universe in order to reduce the size of an $\mathrm{N}$-dimensional asset universe significantly. The starting point for the clustering is the covariance matrix after shrinkage, for which we rely on a constant correlation matrix (set equal to the sample average) as structured estimator (see Ledoit and Wolf, 2004b).

\subsection{Heterogeneity of the Asset Universe}

In order to benefit from diversification when applying portfolio selection rules, the reduced subset $n \subset N$ should consist of the $n$ assets with the lowest overall correlation with each other. Identifying this sub-space can be translated into the problem of finding the longest path of $n \subset N$ vertices in a simple cycle of an undirected graph. The distance between the vertices can be represented by $d_{i, j}=1-\rho_{i, j}$, where $\rho_{i, j}$ is the correlation 
between assets $i$ and $j$. A correlation of 0.8 would then be equal to a Euclidean distance of 0.2 , whereas a correlation of -0.2 would be 1.2 . Unfortunately, due to the curse of dimensionality, analyzing all subspaces of $n \subset N$ is not a feasible task. ${ }^{6}$ Furthermore, from an optimization point of view, the challenge of finding the longest path is a $\mathcal{N} \mathcal{P}$ hard problem, i.e. cannot be solved in polynomial time, and general approximation techniques are not available.

Therefore, we propose a heuristic method, hierarchical clustering, for decomposing a universe of $N$ assets into $n$ subspaces. We then choose the most representative assets in each subspace, identified as the medoid, in order to preserve the heterogeneity of the original asset universe. The reduction of the asset universe should alleviate the parameter estimation problem.

\section{$3.2 \quad$ Hierarchical Clustering}

Classification and cluster analysis are used to group a collection of objects into subcategories/subsets given a chosen criterion. In this work, hierarchical clustering is proposed as a method to establish the relationship between different components in an asset universe.

In general, hierarchical clustering generates a nested sequence of partitions of objects or observations (see Xu and Wunsch, 2005). More specifically, we consider agglomerative hierarchical clustering, which starts by placing each object in its own cluster and then merges these atomic clusters into increasingly larger clusters until all objects are enveloped (see e.g. Tan et al., 2006). Given a set of objects and a clustering criterion, the partition of the objects into clusters is carried out such that the similarity within a given cluster and the dissimilarity among different clusters is maximized. While the dissimilarity between pairs of observations is measured by an appropriate distance metric, a linkage criterion specifies the dissimilarity of clusters as a function of the pairwise distances of the members in each cluster. The choice of an appropriate metric will influence the shape of the clusters, as some elements may be close to one another according to one distance and farther apart according to another distance measure (e.g. the Manhattan distance and the Euclidean distance will indicate different lengths between two points in a 2-dimensional space). Furthermore, there exist several linkage criteria in the literature, where the most commonly used criteria are complete linkage clustering and

\footnotetext{
${ }^{6}$ For example, finding the optimal universe of the least correlated 15 out of 50 assets would require approximately $2.25 \cdot 10^{12}$ permutations.
} 
single linkage clustering:

$$
\begin{array}{ll}
\text { Complete linkage } & \max \{d(a, b): a \in A, b \in B\} \\
\text { Single linkage } & \min \{d(a, b): a \in A, b \in B\},
\end{array}
$$

where $d$ is a distance measure. While in single-linkage clustering the similarity of two clusters is given by the similarity of their most similar members, in complete-linkage clustering the similarity of two clusters is determined by their most dissimilar members. Hence, using different linkage criteria has a large influence on the size and shape of the clusters, and choosing an appropriate distance metric and linkage criteria is therefore crucial when classifying elements in a universe. Single linkage clustering is prone to the so-called chaining phenomenon, where clusters may be forced together due to single elements being close to each other, even though many of the elements in each cluster may be very distant from each other. Complete linkage avoids this drawback and tends to find compact clusters of approximately equal diameters. Therefore, we adopt the method of complete linkage in this paper (for a discussion on single- versus complete linkage see Hartigan, 1981).

As established earlier, correlation is a feasible distance measure. Therefore, agglomerative hierarchical clustering can be used to identify and cluster assets into a hierarchical structure according to their correlation, and a pruning level determines the number of clusters. Although the distance matrix used in the hierarchical clustering has to be estimated, this estimation is only used as a basis for the preliminary coarse grid and not as a direct input parameter in the portfolio optimization, i.e. the problem of parameter uncertainty is less severe. For all cases we use shrinkage to estimate the covariance matrix as proposed by Ledoit and Wolf (2004b), with a constant correlation matrix as structured estimator. In this way, the estimation error is reduced and the requirement of a non-singular matrix is satisfied.

When the overall structure of the universe is established and $n$ groups (also called clusters or sets) are formed, representative assets (so-called pillars) are chosen from each cluster to constitute a reduced asset menu on which the portfolio rules are applied. As the representative asset we use the medoid.

\subsection{Exhibition}

In order to illustrate the proposed technique in a still confined data set, we use the 49 industry portfolios from Kenneth French's website. The 49 industry portfolios are 
composed of stocks traded on the NYSE, AMEX, and NASDAQ according to their fourdigit SIC code. The monthly data span the period January 1970 to July 2013. First, we use the shrinkage technique to compute the $49 \times 49$ correlation matrix and transform it to a Euclidean distance matrix. We then use agglomerative hierarchical clustering with a complete linkage criterion.

The dendrogram in Figure 1 illustrates at which level the different sub-clusters are merged. Portfolios which are highly correlated, i.e. have a small distance to their

Dendrogram of the 49 industry portfolios

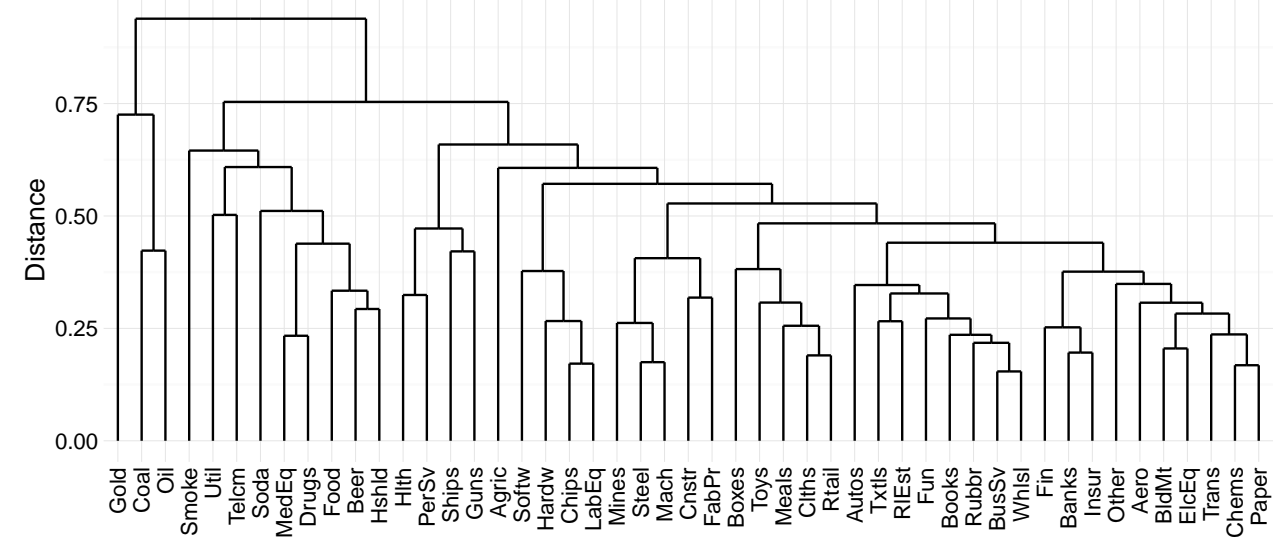

Figure 1: Dendrogram illustrating the correlation structure of the 49 industry portfolios neighbors, are linked together at an early stage. One example is the portfolios Mines, Steel, and Mach. The three portfolios denote the mining, the steel, and the machinery industries, respectively, which due to their business sectors are highly interconnected. The tree structure of the dendrogram can be exploited to form groups of assets. By pruning at specific levels of the tree, a desired number of sets can be constructed. For the purely illustrative purpose here, we decide to reduce the universe to $n=4$. The result of maximizing the inter-cluster dissimilarity and the intra-cluster similarity can be visualized with a principal component analysis (PCA). Figure 2 shows the convex hull of each cluster projected on the first two principal components. Furthermore, their corresponding pillars are indicated with a black bullet. It can be seen that the portfolios are not evenly distributed across the different clusters. Set 1 holds a particularly large amount of portfolios, while set 3 is a single portfolio. By projecting the 49 dimensions of this example onto the first two principal components, the areas of sets 1 and 2 overlap. Of course, in the multidimensional space the convex hulls of the two sets do not overlap. 


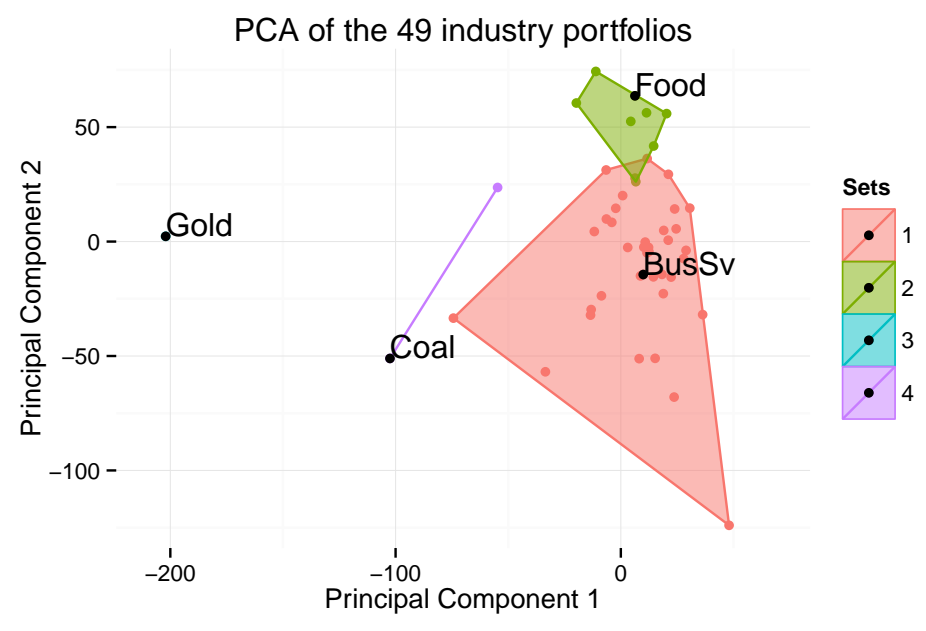

Figure 2: First two principal components of the 49 industry portfolios divided into 4 groups according to hierarchical clustering with complete linkage. The black markers indicate the pillar of each group with the corresponding name.

The reduction of the 49 industry portfolios results in the following asset menu: Business Services (BusSv), Food, Coal, and Gold. ${ }^{7}$ The maximum, minimum and average correlations of the portfolios in both universes are summarized in Table 1.

\begin{tabular}{ccc} 
& Full universe & Reduced universe \\
\hline Max & 0.86 & 0.63 \\
Min & 0.06 & 0.12 \\
Average & 0.57 & 0.35 \\
\hline
\end{tabular}

Table 1: Maximum, minimum and average correlation of the 49 industry portfolios, and the reduced universe of four portfolios.

As expected, the maximum and average correlation of the reduced universe has decreased considerably compared to the full universe as only one pillar represents all members of a cluster (which by construction have a high within-group correlation).

\section{Results}

This section assesses the performance of the minimum-variance portfolio (MVP), the mean-variance tangency portfolio (TP), the three-fund rule (KZ), the four-fund rule

\footnotetext{
${ }^{7}$ The extreme reduction of dimensionality in this exhibition is used for the illustration purpose only.
} 
(TZ), and the $1 / \mathrm{N}$ portfolio $(1 / \mathrm{N})$ for the full and the reduced asset universes. The use of the feature selection technique is denoted by FS. The analysis is based on monthly returns of two data sets: (a) the 49 industry portfolios and (b) the constituent stocks of the S\&P 500 from January 1970 to July 2013. We conduct an out-of-sample back-test where only data up to the time of the portfolio choice are used. The returns of the different portfolio selection rules are determined by the realized returns of the chosen assets one month later when the portfolio is readjusted. The reduced asset universe is constructed at the beginning of every year. Given that the focus is on illustrating the benefit of feature selection and not on making a horse race between the different portfolio selection rules, we deliberately do not account for transaction costs and use gross returns. However, we indicate the portfolio turnover on an annual basis. Furthermore, for the parameter estimation we use a "rolling window" with a length of either 60, 120, 180 or 240 months.

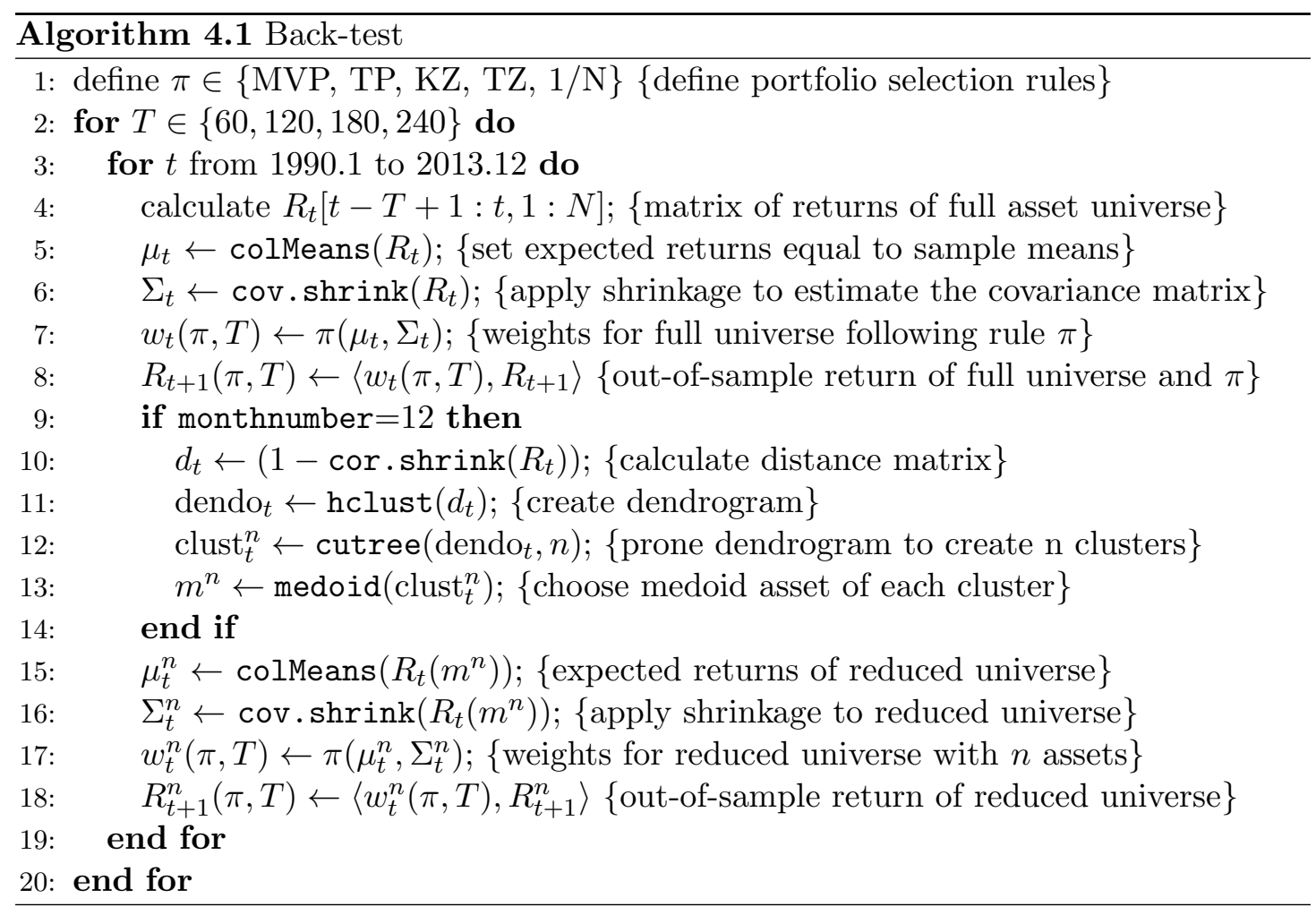

In Algorithm 4.1 we describe precisely how the back-test and the clustering is implemented in the $\mathrm{R}$ programming language. In addition to the standard libraries, we use also the tawny package for the shrinkage technique (see Ledoit and Wolf, 2003). For each chosen strategy and observation period, we first calculate monthly returns. We 
use them to compute the sample means and apply shrinkage to estimate the covariance matrix on the full asset menu (lines 4-6). ${ }^{8}$ Then, for optimal portfolios according to the different selection rules, we calculate an out-of-sample return over the next month (lines 7-8). The Euclidean scalar product is denoted by $\langle\cdot, \cdot\rangle$. At the end of each year we choose representative assets for the next year. ${ }^{9}$ Therefore, in line 10 , we calculate the distance matrix (distance measure is equal to one minus the correlation) that is used by the clustering algorithm. In lines 11 and 12, we calculate the dendrogram of the full asset universe and prune the tree to obtain $n$ clusters. Throughout our calculations we have used exactly 15 clusters for both the 49 industry portfolios and the S\&P 500. Then, we choose the medoid to be the representative asset in each cluster, see line 13. Lines 15-18 repeat the operations 5-8 on the reduced asset menu. Our back-test was run on a machine with Intel core i5 (2.53 GHz, 3MB L3 cache) and 8 GB RAM. For the S\&P 500 data set, all calculations of the back-test can be conducted in less than one hour. ${ }^{10}$

60 months for parameter estimation

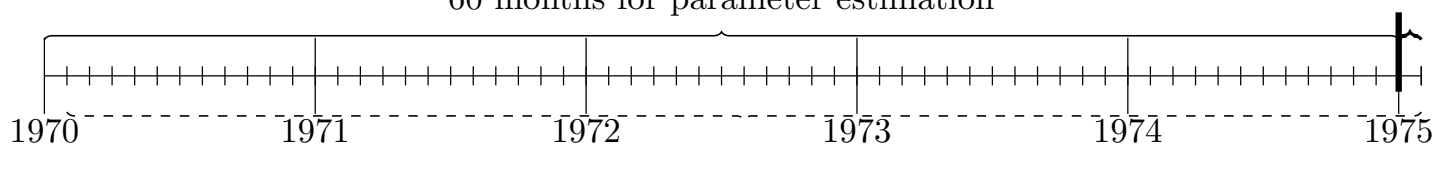

Figure 3: Example for the back-test approach (with an estimation window of 60 months).

Figure 3 shows the back-test approach for a rolling window of 60 months (solid brace) at the end of year 1974, when parameters are estimated for the first time and when the portfolio is optimized according to the different portfolio selection rules. One month later the return of the chosen portfolio is measured and the time window for parameter estimation is shifted by one month (dashed brace). Given that we use all available data for all the different observation periods, the number of out-of-sample returns differs. While, e.g., for an estimation window of 60 months 463 out-of-sample returns can be calculated, this number reduces to 283 in case of a 240 months window. Furthermore, in order to avoid that our performance comparisons are determined by a specific inception date, in addition to the assessment over the whole period we also evaluate realized returns of the different portfolio rules over consecutive 10 year periods (shifted by one year). This iterative testing of the portfolio rules is applied until the back-test ends in

\footnotetext{
${ }^{8}$ The structured estimator is based on the constant correlation matrix (set equal to the average sample correlation), which corresponds to the default value in the package.

${ }^{9}$ Given that the clusters are quite stable over time and the assets within a cluster are highly correlated, in order to reduce excessive trading we propose to apply feature selection on an annual basis.

${ }^{10}$ The most time consuming operation is shrinkage. For the 49 industry portfolios the computational time for all results is less than 10 minutes.
} 
the year 2013. This means that each portfolio rule is evaluated repeatedly on each data set (with and without feature selection) and with each of the four rolling windows.

The performance assessment of the different portfolio rules is based on annualized alpha. Alpha represents the return of a strategy beyond what would be expected given the exposure to the relevant risk factors (for which a corresponding risk premium should be earned). While the Capital Asset Pricing Model (CAPM) implies that the excess return of the market portfolio ( $E X M K T$ ) over the risk-free rate $r$ is the only explaining risk factor, the Arbitrage Pricing Theory provides the theoretical foundation for including arbitrary (additional) risk factors beyond the market portfolio. Fama and French (1993), for short FF, identified empirically additional return-predicting risk factors: the excess return on a portfolio of small stocks over a portfolio of large stocks $(S M B)$ and the excess return on a portfolio of high book-to-market stocks over a portfolio of low book-to-market stocks $(H M L)$. Carhart (1997), short FFC, shows that in addition to the three Fama-French factors an additional fourth predictor, the momentum factor $(U M D)$, should be considered. Momentum in a stock is described as the tendency for the stock price to continue rising if it is going up and to continue declining if it is going down. The $U M D$ can be calculated by subtracting the equally weighted average of the highest performing firms from the equally weighted average of the lowest performing firms, lagged by one month. Specifically, we conduct the following regressions

$$
R_{p, t}-r_{t}=\alpha+\sum_{j} F_{j, t} \beta_{j}+\epsilon_{t}
$$

with $F_{j, t} \in\left\{E X M K T_{t}\right\}$ for the CAPM model, $F_{j, t} \in\left\{E X M K T_{t}, S M B_{t}, H M L_{t}\right\}$ for the FF model, and $F_{j, t} \in\left\{E X M K T_{t}, S M B_{t}, H M L_{t}, U M D_{t}\right\}$ for the FFC model. The time series of all risk factors are available on Kenneth French's website.

Finally, in order to measure whether feature selection improves alpha significantly, we create long/short portfolios (LS). Specifically, for each of the different test cases we take a long position in the optimal portfolio of the reduced asset universe and a short position in that of the full universe.

\subsection{The 49 Industry Portfolios}

The data are collected from the Kenneth French data library. We use monthly valueweighted returns of each industry portfolio from January 1970 to July 2013. Table 2 shows the annualized alpha for each portfolio rule and estimation window over the whole back-test period, and Table 3 gives the corresponding annual portfolio turnover. 


\begin{tabular}{|c|c|c|c|c|c|c|c|c|c|c|c|c|}
\hline & \multicolumn{3}{|c|}{ window $=60$} & \multicolumn{3}{|c|}{ window $=120$} & \multicolumn{3}{|c|}{ window $=180$} & \multicolumn{3}{|c|}{ window $=240$} \\
\hline & CAPM & $\mathrm{FF}$ & FFC & CAPM & $\mathrm{FF}$ & FFC & CAPM & $\mathrm{FF}$ & FFC & CAPM & $\mathrm{FF}$ & FFC \\
\hline \multirow[t]{2}{*}{ MVP } & 1.22 & -0.58 & 0.11 & 0.73 & -0.75 & 0.00 & 0.71 & -0.62 & 0.11 & 0.99 & -0.64 & 0.16 \\
\hline & $(0.15)$ & $(0.43)$ & $(0.88)$ & $(0.43)$ & $(0.34)$ & $(1.00)$ & $(0.48)$ & $(0.45)$ & $(0.89)$ & $(0.38)$ & $(0.47)$ & $(0.85)$ \\
\hline \multirow[t]{2}{*}{$\mathrm{MVP}+\mathrm{FS}$} & 0.69 & -1.07 & -1.17 & 1.51 & -0.08 & 0.17 & 2.62 & 1.53 & 1.65 & 2.88 & 1.13 & 1.44 \\
\hline & $(0.56)$ & $(0.33)$ & $(0.30)$ & $(0.25)$ & $(0.95)$ & $(0.89)$ & $(0.04)$ & $(0.21)$ & $(0.19)$ & $(0.08)$ & $(0.44)$ & $(0.34)$ \\
\hline \multirow[t]{2}{*}{$\mathrm{MVP}+\mathrm{LS}$} & -0.53 & -0.49 & -1.28 & 0.78 & 0.67 & 0.18 & 1.90 & 2.15 & 1.53 & 1.89 & 1.77 & 1.28 \\
\hline & $(0.50)$ & $(0.53)$ & $(0.10)$ & $(0.35)$ & $(0.43)$ & $(0.84)$ & $(0.03)$ & $(0.01)$ & $(0.08)$ & $(0.05)$ & $(0.03)$ & $(0.14)$ \\
\hline \multirow[t]{2}{*}{$\mathrm{TP}$} & 1.17 & -0.52 & 0.30 & 0.62 & -0.76 & -0.04 & 0.65 & -0.66 & 0.06 & 0.95 & -0.67 & 0.12 \\
\hline & $(0.19)$ & $(0.51)$ & $(0.70)$ & $(0.49)$ & $(0.34)$ & $(0.96)$ & $(0.52)$ & $(0.43)$ & $(0.94)$ & $(0.40)$ & $(0.45)$ & $(0.88)$ \\
\hline \multirow[t]{2}{*}{$\mathrm{TP}+\mathrm{FS}$} & 4.26 & 4.21 & 5.26 & 1.25 & -0.13 & -0.11 & 2.46 & 1.39 & 1.43 & 2.66 & 0.95 & 1.25 \\
\hline & $(0.13)$ & $(0.14)$ & $(0.07)$ & $(0.36)$ & $(0.92)$ & $(0.94)$ & $(0.06)$ & $(0.27)$ & $(0.26)$ & $(0.11)$ & $(0.53)$ & $(0.41)$ \\
\hline \multirow[t]{2}{*}{$\mathrm{TP}+\mathrm{LS}$} & 3.10 & 4.73 & 4.96 & 0.63 & 0.62 & -0.07 & 1.81 & 2.05 & 1.37 & 1.71 & 1.62 & 1.13 \\
\hline & $(0.25)$ & $(0.08)$ & $(0.07)$ & $(0.50)$ & $(0.51)$ & $(0.94)$ & $(0.05)$ & $(0.02)$ & $(0.13)$ & $(0.16)$ & $(0.20)$ & $(0.40)$ \\
\hline \multirow[t]{2}{*}{$\mathrm{KZ}$} & 2.39 & 2.54 & 1.28 & 2.89 & 2.37 & 1.40 & 2.78 & 2.58 & 1.49 & 1.56 & 1.66 & 0.82 \\
\hline & $(0.25)$ & $(0.22)$ & $(0.54)$ & $(0.10)$ & $(0.18)$ & $(0.43)$ & $(0.12)$ & $(0.14)$ & $(0.40)$ & $(0.46)$ & $(0.43)$ & (0.69) \\
\hline \multirow[t]{2}{*}{$\mathrm{KZ}+\mathrm{FS}$} & 1.86 & 1.96 & -0.19 & 4.32 & 3.50 & 2.52 & 2.91 & 1.98 & 1.06 & 1.58 & 0.78 & -0.16 \\
\hline & $(0.27)$ & $(0.25)$ & $(0.91)$ & $(0.01)$ & $(0.02)$ & $(0.10)$ & $(0.08)$ & $(0.21)$ & $(0.50)$ & $(0.43)$ & $(0.69)$ & $(0.93)$ \\
\hline \multirow[t]{2}{*}{$\mathrm{KZ}+\mathrm{LS}$} & -0.54 & -0.58 & -1.47 & 1.44 & 1.13 & 1.12 & 0.14 & -0.60 & -0.43 & -0.02 & -0.88 & -0.98 \\
\hline & $(0.76)$ & $(0.74)$ & $(0.42)$ & $(0.33)$ & $(0.45)$ & $(0.47)$ & $(0.92)$ & $(0.67)$ & $(0.77)$ & $(0.92)$ & $(0.50)$ & $(0.48)$ \\
\hline \multirow[t]{2}{*}{$\mathrm{TZ}$} & 12.81 & 2.91 & 25.75 & 2.00 & 1.07 & 0.74 & 2.11 & 1.55 & 0.93 & 1.32 & 1.00 & 0.50 \\
\hline & $(0.41)$ & $(0.85)$ & $(0.08)$ & $(0.05)$ & $(0.28)$ & $(0.46)$ & $(0.09)$ & $(0.20)$ & $(0.44)$ & $(0.41)$ & $(0.53)$ & $(0.75)$ \\
\hline \multirow[t]{2}{*}{$\mathrm{TZ}+\mathrm{FS}$} & 3.96 & -0.72 & 0.88 & 3.85 & 2.88 & 2.11 & 2.96 & 1.96 & 1.12 & 1.59 & 0.69 & -0.20 \\
\hline & $(0.41)$ & $(0.88)$ & $(0.95)$ & $(0.01)$ & $(0.03)$ & $(0.12)$ & $(0.05)$ & $(0.17)$ & $(0.44)$ & $(0.70)$ & $(0.91)$ & (0.69) \\
\hline \multirow[t]{2}{*}{$\mathrm{TZ}+\mathrm{LS}$} & -8.84 & -3.63 & -26.06 & 1.85 & 1.80 & 1.37 & 0.85 & 0.41 & 0.19 & 0.27 & -0.31 & -0.70 \\
\hline & $(0.60)$ & $(0.83)$ & $(0.11)$ & $(0.09)$ & $(0.10)$ & $(0.21)$ & $(0.45)$ & $(0.71)$ & $(0.87)$ & $(0.95)$ & $(0.71)$ & $(0.53)$ \\
\hline \multirow[t]{2}{*}{$1 / \mathrm{N}$} & 1.38 & -0.33 & 0.14 & 1.04 & -0.41 & 0.15 & 1.07 & -0.25 & 0.31 & 1.29 & -0.30 & 0.37 \\
\hline & $(0.08)$ & $(0.62)$ & $(0.83)$ & $(0.22)$ & $(0.58)$ & $(0.84)$ & $(0.26)$ & $(0.75)$ & $(0.68)$ & $(0.24)$ & $(0.72)$ & (0.66) \\
\hline \multirow[t]{2}{*}{$1 / \mathrm{N}+\mathrm{FS}$} & 1.20 & -0.35 & -0.56 & 1.76 & 0.28 & 0.44 & 2.71 & 1.57 & 1.57 & 3.06 & 1.35 & 1.55 \\
\hline & $(0.23)$ & $(0.71)$ & $(0.55)$ & $(0.13)$ & $(0.80)$ & $(0.69)$ & $(0.03)$ & $(0.17)$ & $(0.18)$ & $(0.05)$ & $(0.32)$ & $(0.26)$ \\
\hline \multirow[t]{2}{*}{$1 / \mathrm{N}+\mathrm{LS}$} & -0.19 & -0.01 & -0.70 & 0.72 & 0.68 & 0.29 & 1.63 & 1.81 & 1.25 & 1.77 & 1.77 & 0.64 \\
\hline & $(0.77)$ & $(0.98)$ & $(0.27)$ & $(0.31)$ & $(0.34)$ & $(0.68)$ & $(0.03)$ & $(0.02)$ & $(0.10)$ & $(0.10)$ & $(0.13)$ & $(0.30)$ \\
\hline
\end{tabular}

Table 2: Fama-French 49 Industry portfolios: $\alpha$ per annum for different models. The $p$-values are shown in brackets.

The results can be summarized as follows: For the short estimation window of 60 months, in line with Kritzman (1993), the results are mixed, and no portfolio rule (with and without feature selection) has a statistically significant positive alpha. At the same time we observe an extreme portfolio turnover for the Kan and Zhou (2007) and Tu and Zhou (2011) models.

For longer periods ( $\geq 120$ months), in most cases results of the reduced universe outperform those of the full universe, and some of the alphas turn out to be statistically significant relative to the CAPM and the FF model. The same can be observed also for the LS strategy. Given that due to our choice of using all available data the out-ofsample returns cover different time intervals (see discussion above), here we deliberately abstain from comparing row-wise alphas of one specific portfolio selection rule in order to 


\begin{tabular}{|c|c|c|c|c|c|c|c|c|c|c|c|c|c|c|c|}
\hline \multirow[b]{2}{*}{$T$} & \multicolumn{5}{|c|}{ Without Feature Selection } & \multicolumn{5}{|c|}{ With Feature Selection } & \multicolumn{5}{|c|}{ Long/Short } \\
\hline & MVP & $\mathrm{TP}$ & $\mathrm{KZ}$ & $\mathrm{TZ}$ & $1 / \mathrm{N}$ & MVP & $\mathrm{TP}$ & $\mathrm{KZ}$ & $\mathrm{TZ}$ & $1 / \mathrm{N}$ & MVP & $\mathrm{TP}$ & $\mathrm{KZ}$ & $\mathrm{TZ}$ & $1 / \mathrm{N}$ \\
\hline 60 & 66.0 & 102.1 & 1033.1 & 458.5 & 38.2 & 46.9 & 87.6 & 179.8 & 148.7 & 42.7 & 184.6 & 243.4 & 1236.5 & 849.2 & 55.1 \\
\hline 120 & 62.6 & 63.5 & 581.8 & 315.6 & 39.1 & 41.1 & 42.0 & 124.0 & 102.3 & 44.5 & 161.9 & 188.3 & 692.3 & 450.5 & 56.8 \\
\hline 180 & 61.2 & 61.3 & 442.6 & 304.7 & 39.5 & 38.3 & 38.7 & 98.5 & 87.6 & 45.5 & 153.0 & 179.3 & 542.4 & 417.7 & 57.4 \\
\hline 240 & 61.1 & 61.1 & 408.5 & 307.5 & 40.9 & 33.7 & 34.5 & 70.5 & 64.5 & 49.3 & 145.6 & 170.1 & 472.0 & 385.8 & 60.4 \\
\hline
\end{tabular}

Table 3: 49 industry portfolios: Average annual turnover in percentage over the complete back-test period.

find the optimal observation period. ${ }^{11}$ The indicated turnover for the portfolio selection rules with feature selection considers the monthly readjustment of the portfolio weights as well as the annual selection of new representative assets. To summarize, with the exception of $1 / \mathrm{N}$ (where results are similar), applying feature selection on an annual basis lowers the portfolio turnover. For the Kan and Zhou (2007) and Tu and Zhou (2011) models this reduction in turnover is remarkable.

Interestingly, we observe this improvement also for the $1 / \mathrm{N}$ rule, which is normally hard to outperform (see e.g. DeMiguel et al., 2009b). We explain this finding by the avoidance of sector concentration together with choosing low-beta assets. As a support to this argument, in Table 4 we indicate the beta of the $1 / \mathrm{N}$ rule for the whole and the reduced universes. After selecting only the representative pillars of each cluster, the beta declines considerably. In this way feature selection relates to the well documented phenomenon of "betting against beta" of Frazzini and Pedersen (2014).

\begin{tabular}{ccc}
\hline & $1 / \mathrm{N}$ & $1 / \mathrm{N}+\mathrm{FS}$ \\
\hline 60 months & 1.03 & 0.95 \\
120 months & 1.01 & 0.93 \\
180 months & 1.00 & 0.89 \\
240 months & 0.98 & 0.90 \\
\hline
\end{tabular}

Table 4: 49 Industry portfolios: $\beta$ of the $1 / \mathrm{N}$ rule; with and without feature selection.

As a robustness check, we divide the out-of-sample back-test period into consecutive 10-year periods (the number depends on the observation period) to check whether the outperformance using feature selection is driven by a few sub-periods with a very large alpha. Table 5 reports the percentage of 10-year periods in which alpha improves after using feature selection. It is noteworthy that the majority of test cases show a percentage well above $50 \%$, i.e. alpha increases after applying feature selection. Again, in line

\footnotetext{
${ }^{11}$ When considering only out-of-sample returns of the same time-interval, we found that intermediate estimations windows of 10-20 years perform best. Results are available upon request.
} 
with previous results, most optimization rules with feature selection benefit from longer observation periods.

\begin{tabular}{|c|c|c|c|c|c|c|c|c|c|c|c|c|}
\hline & \multicolumn{3}{|c|}{ window $=60$} & \multicolumn{3}{|c|}{ window $=120$} & \multicolumn{3}{|c|}{ window $=180$} & \multicolumn{3}{|c|}{ window $=240$} \\
\hline & CAPM & $\mathrm{FF}$ & FFC & CAPM & $\mathrm{FF}$ & FFC & CAPM & $\mathrm{FF}$ & FFC & CAPM & $\mathrm{FF}$ & FFC \\
\hline MVP & 46.4 & 32.1 & 10.7 & 47.8 & 47.8 & 52.2 & 88.9 & 88.9 & 77.8 & 100.0 & 100.0 & 100.0 \\
\hline $\mathrm{TP}$ & 39.3 & 32.1 & 17.9 & 47.8 & 47.8 & 47.8 & 88.9 & 88.9 & 72.2 & 100.0 & 100.0 & 100.0 \\
\hline $\mathrm{KZ}$ & 64.3 & 64.3 & 39.3 & 82.6 & 78.3 & 78.3 & 66.7 & 50.0 & 33.3 & 76.9 & 15.4 & 15.4 \\
\hline $\mathrm{TZ}$ & 50.0 & 35.7 & 25.0 & 91.3 & 82.6 & 73.9 & 77.8 & 61.1 & 33.3 & 69.2 & 30.8 & 30.8 \\
\hline $1 / \mathrm{N}$ & 53.6 & 39.3 & 21.4 & 52.2 & 43.5 & 47.8 & 94.4 & 88.9 & 66.7 & 100.0 & 100.0 & 92.3 \\
\hline
\end{tabular}

Table 5: 49 industry portfolios: Percentage of 10-year intervals for which the alpha of a specific test case benefits from reducing the asset universe by feature selection.

\subsection{The S\&P 500 Universe}

The Standard \& Poor's 500 Index covers $70 \%$ of the overall U.S. market capitalization. It consists of 500 large US companies listed at the NYSE and NASDAQ. We consider the index composition and the corresponding companies during the period January 1970 to July 2013. If a company leaves the index while it is held in a portfolio, we sell it at the next (monthly) re-adjustment stage.

In addition to be less prone to outliers, shrinkage avoids the problem of a singular sample covariance matrix in case of more assets than observations. For the full S\&P 500 asset universe more than 41.6 years of monthly observations are required to prevent singularity. However, only a few companies have been members of the index for such a long period of time. Furthermore, even if the data were available, due to possible time-varying parameters it is not clear if these old observations are relevant, and if they should be used. Hence, also for this data set we use the shrinkage technique proposed by Ledoit and Wolf (2004b) to calculate the correlation matrix required for the hierarchical clustering.

In order to determine the weight of the global minimum variance portfolio and of the tangency portfolio, Kan and Zhou (2007) and Tu and Zhou (2011) use an adjusted estimator for the true Sharpe ratio. Therefore, their approach relies on the incomplete beta function, which is defined as

$$
B_{z}(a, b)=\int_{0}^{z} t^{a-1}(1-t)^{b-1} d t,
$$

where $0 \leq z \leq 1, a=N / 2$ and $b=(T-N) / 2$. For more assets than observations 
$(T<N)$ the incomplete beta function is not defined, i.e. it is not possible to use these portfolio selection rules. Therefore, as an additional contribution, feature selection allows to apply these rules on large data sets.

\begin{tabular}{|c|c|c|c|c|c|c|c|c|c|c|c|c|}
\hline & \multicolumn{3}{|c|}{ window $=60$} & \multicolumn{3}{|c|}{ window $=120$} & \multicolumn{3}{|c|}{ window $=180$} & \multicolumn{3}{|c|}{ window $=240$} \\
\hline & CAPM & $\mathrm{FF}$ & FFC & CAPM & $\mathrm{FF}$ & FFC & CAPM & $\mathrm{FF}$ & FFC & CAPM & $\mathrm{FF}$ & FFC \\
\hline \multirow[t]{2}{*}{ MVP } & 1.02 & -1.07 & 0.95 & 0.68 & -1.14 & 0.23 & 0.67 & -1.14 & 0.42 & 2.18 & 0.04 & 1.65 \\
\hline & $(0.32)$ & $(0.24)$ & $(0.22)$ & $(0.53)$ & $(0.23)$ & $(0.45)$ & $(0.60)$ & $(0.27)$ & $(0.66)$ & $(0.38)$ & $(0.46)$ & $(0.77)$ \\
\hline \multirow[t]{2}{*}{$\mathrm{MVP}+\mathrm{FS}$} & 0.95 & -0.80 & 2.01 & 2.91 & 1.12 & 2.79 & 2.37 & 0.82 & 2.01 & 4.53 & 2.66 & 4.63 \\
\hline & $(0.57)$ & $(0.62)$ & $(0.18)$ & $(0.07)$ & $(0.47)$ & $(0.16)$ & $(0.60)$ & $(0.20)$ & $(0.56)$ & $(0.02)$ & $(0.14)$ & $(0.01)$ \\
\hline \multirow[t]{2}{*}{$\mathrm{MVP}+\mathrm{LS}$} & -0.07 & 0.27 & 0.06 & 0.97 & 0.99 & 1.41 & 3.15 & 3.38 & 3.99 & 2.35 & 2.62 & 2.98 \\
\hline & $(0.95)$ & $(0.83)$ & $(0.40)$ & $(0.46)$ & $(0.46)$ & $(0.30)$ & $(0.02)$ & $(0.01)$ & $(0.00)$ & $(0.05)$ & $(0.04)$ & $(0.02)$ \\
\hline \multirow[t]{2}{*}{$\mathrm{TP}$} & 0.63 & -1.04 & 1.33 & 0.31 & -1.26 & 0.63 & 0.52 & -1.22 & 0.36 & 2.11 & -0.01 & 1.60 \\
\hline & $(0.56)$ & $(0.30)$ & $(0.12)$ & $(0.77)$ & $(0.19)$ & $(0.45)$ & $(0.68)$ & $(0.25)$ & $(0.71)$ & $(0.33)$ & $(0.45)$ & $(0.88)$ \\
\hline \multirow[t]{2}{*}{$\mathrm{TP}+\mathrm{FS}$} & -0.67 & -0.86 & 2.31 & 0.63 & -0.69 & 1.47 & 3.49 & 2.26 & 4.24 & 4.52 & 2.79 & 4.80 \\
\hline & $(0.78)$ & $(0.72)$ & $(0.32)$ & $(0.71)$ & $(0.68)$ & $(0.36)$ & $(0.04)$ & $(0.18)$ & $(0.01)$ & $(0.02)$ & $(0.13)$ & $(0.01)$ \\
\hline \multirow[t]{2}{*}{$\mathrm{TP}+\mathrm{LS}$} & -1.29 & 0.18 & 0.98 & 0.30 & 0.54 & 0.82 & 2.96 & 3.45 & 3.87 & 2.41 & 2.80 & 3.20 \\
\hline & $(0.55)$ & $(0.93)$ & $(0.66)$ & $(0.82)$ & $(0.69)$ & $(0.55)$ & $(0.04)$ & $(0.02)$ & $(0.01)$ & $(0.06)$ & $(0.03)$ & $(0.02)$ \\
\hline \multirow[t]{2}{*}{$\mathrm{KZ}+\mathrm{FS}$} & 1.44 & 0.92 & -0.03 & 0.22 & -1.15 & -1.11 & 1.82 & 0.69 & 0.46 & 2.82 & 1.33 & 1.19 \\
\hline & $(0.46)$ & $(0.63)$ & $(0.99)$ & $(0.90)$ & $(0.49)$ & $(0.51)$ & $(0.34)$ & $(0.69)$ & $(0.79)$ & $(0.21)$ & $(0.48)$ & $(0.54)$ \\
\hline \multirow[t]{2}{*}{$\mathrm{TZ}+\mathrm{FS}$} & 1.38 & 0.68 & 0.46 & 0.37 & -1.03 & -0.70 & 1.99 & 0.71 & 0.85 & 2.90 & 1.31 & 1.40 \\
\hline & $(0.38)$ & $(0.66)$ & $(0.77)$ & $(0.80)$ & $(0.47)$ & $(0.63)$ & $(0.25)$ & $(0.64)$ & $(0.58)$ & $(0.16)$ & $(0.44)$ & $(0.42)$ \\
\hline \multirow[t]{2}{*}{$1 / \mathrm{N}$} & 1.50 & -0.48 & 0.91 & 1.24 & -0.48 & 0.73 & 1.19 & -0.59 & 0.60 & 2.07 & 0.47 & 2.06 \\
\hline & $(0.08)$ & $(0.51)$ & $(0.16)$ & $(0.19)$ & $(0.48)$ & $(0.32)$ & $(0.30)$ & $(0.51)$ & $(0.48)$ & $(0.24)$ & $(0.72)$ & $(0.66)$ \\
\hline \multirow[t]{2}{*}{$1 / \mathrm{N}+\mathrm{FS}$} & 1.55 & -0.37 & 1.46 & 1.50 & -0.43 & 1.36 & 3.55 & 1.72 & 3.57 & 3.82 & 1.78 & 3.38 \\
\hline & $(0.26)$ & $(0.78)$ & $(0.24)$ & $(0.32)$ & $(0.76)$ & $(0.32)$ & $(0.03)$ & $(0.23)$ & $(0.01)$ & $(0.04)$ & $(0.23)$ & $(0.02)$ \\
\hline \multirow[t]{2}{*}{$1 / \mathrm{N}+\mathrm{LS}$} & 0.05 & 0.11 & 0.55 & 0.25 & 0.13 & 0.62 & 2.36 & 2.30 & 2.97 & 1.75 & 1.31 & 1.32 \\
\hline & $(0.96)$ & $(0.91)$ & $(0.60)$ & $(0.82)$ & $(0.91)$ & $(0.58)$ & $(0.04)$ & $(0.04)$ & $(0.01)$ & $(0.13)$ & $(0.15)$ & $(0.09)$ \\
\hline
\end{tabular}

Table 6: S\&P500: $\alpha$ per annum for different models. The $p$-values are shown in brackets.

Table 6 reports the annualized alpha of the different portfolio selection rules over the whole period. In line with the 49 industry data set, no portfolio selection rule generates a significant positive alpha on the whole universe, and we observe a consistent improvement with feature selection. Also for this data set the different models benefit clearly from longer estimation periods, especially for the reduced asset universe. Most LS strategies perform best with a 180 or 240 months observation period, and some are significant with respect to the different factor models. Table 7 shows the corresponding annualized turnover. For this data set, feature selection applied on an annual basis reduces trading costs by more than $100 \%$, and therefore mitigates the problem of excessive trading.

Again, the improvement of feature selection holds also for the $1 / \mathrm{N}$ rule, which we attribute to the reduced concentration risk and low-beta stocks. To support this explanation, in Table 8 we show the beta of the $1 / \mathrm{N}$ portfolios for the whole and the reduced universe. We observe that after selecting only the representative pillars of each cluster, beta declined for longer observation periods. However, for shorter observation periods 


\begin{tabular}{|c|c|c|c|c|c|c|c|c|c|c|c|c|c|c|c|}
\hline \multirow[b]{2}{*}{$T$} & \multicolumn{5}{|c|}{ Without Feature Selection } & \multicolumn{5}{|c|}{ With Feature Selection } & \multicolumn{5}{|c|}{ Long/Short } \\
\hline & MVP & $\mathrm{TP}$ & $\mathrm{KZ}$ & $\mathrm{TZ}$ & $1 / \mathrm{N}$ & MVP & $\mathrm{TP}$ & $\mathrm{KZ}$ & $\mathrm{TZ}$ & $1 / \mathrm{N}$ & MVP & $\mathrm{TP}$ & $\mathrm{KZ}$ & $\mathrm{TZ}$ & $1 / \mathrm{N}$ \\
\hline 60 & 313.0 & 356.1 & & & 71.2 & 130.6 & 152.3 & 173.9 & 141.6 & 69.4 & 427.9 & 501.3 & & & 136.0 \\
\hline 120 & 260.5 & 285.0 & & & 71.0 & 117.2 & 120.0 & 132.7 & 117.0 & 68.1 & 364.9 & 391.2 & & & 134.1 \\
\hline 180 & 234.9 & 252.1 & & & 69.0 & 109.2 & 113.0 & 118.5 & 107.7 & 65.9 & 332.4 & 353.7 & & & 129.4 \\
\hline 240 & 202.6 & 211.8 & & & 68.5 & 90.3 & 93.1 & 92.6 & 87.4 & 63.7 & 282.9 & 294.5 & & & 125.9 \\
\hline
\end{tabular}

Table 7: S\&P500: Average annual turnover in percentage over the complete back-test period.

we could not observe this reduction in beta. Given the higher number of individual assets (compared to the 49 portfolios in the previous data set), we attribute this fact to a more severe parameter estimation problem. A more thorough investigation of the effect of feature selection on the beta of a portfolio is left to future research.

\begin{tabular}{ccc}
\hline & $1 / \mathrm{N}$ & $1 / \mathrm{N}+\mathrm{FS}$ \\
\hline 60 months & 1.06 & 1.07 \\
120 months & 1.02 & 1.02 \\
180 months & 1.00 & 0.97 \\
240 months & 0.99 & 0.95
\end{tabular}

Table 8: S\&P500: $\beta$ of the 1/N rule; with and without feature selection

\begin{tabular}{|c|c|c|c|c|c|c|c|c|c|c|c|c|}
\hline & \multicolumn{3}{|c|}{ window $=60$} & \multicolumn{3}{|c|}{ window $=120$} & \multicolumn{3}{|c|}{ window $=180$} & \multicolumn{3}{|c|}{ window $=240$} \\
\hline & CAPM & $\mathrm{FF}$ & FFC & CAPM & $\mathrm{FF}$ & FFC & CAPM & $\mathrm{FF}$ & FFC & CAPM & $\mathrm{FF}$ & FFC \\
\hline MVP & 50.0 & 60.7 & 78.6 & 65.2 & 78.3 & 78.3 & 100 & 94.4 & 100 & 92.3 & 84.6 & 84.6 \\
\hline $\mathrm{TP}$ & 57.1 & 57.2 & 75.0 & 65.2 & 78.3 & 78.3 & 100 & 94.4 & 100 & 92.6 & 84.6 & 84.6 \\
\hline $1 / \mathrm{N}$ & 64.3 & 64.3 & 75.0 & 60.9 & 78.3 & 52.2 & 100 & 94.4 & 94.4 & 84.6 & 76.6 & 76.6 \\
\hline
\end{tabular}

Table 9: S\&P500: Percentage of 10-year intervals for which the alpha of a specific test case benefits from reducing the asset universe by feature selection.

Table 9 shows the percentage of 10-year periods in which alpha increases with feature selection. Here we can clearly see that longer estimation periods improve the results of feature selection, especially for 15 and 20 years.

\section{Conclusion}

Parameter uncertainty is a major cause for the poor out-of-sample performance of portfolio selection rules based on the sample mean and the sample covariance matrix. We 
propose reducing the asset universe with hierarchical clustering before applying the portfolio selection rule. To assess the benefits of our proposal with out-of-sample back-tests, we apply five well-established portfolio selection rules with different estimation windows on two different data sets: the Fama-French 49 industry portfolios and the constituents of the S\&P 500 index. For most test cases, alpha relative to different prominent factor models is improved by using feature selection, and some alphas are statistically significant. We apply a robustness check to show that our results are not driven by a couple of return outliers. Furthermore, in some cases with longer estimation windows also the alpha of a long/short strategy turns out to be statistically significant. We consider this finding to be in support of the proposed approach. Finally, our method mitigates the problem of excessive portfolio turnover.

\section{Acknowledgements}

We thank Michael Hanke, Kourosh Marjani Rasmussen, the Guest Editors Nalan Gulpinar, Xuan V. Doan and Arne K. Strauss, and two anonymous referees for helpful comments. Special thanks also to conference participants of the APMOD 2014 at the University of Warwick and seminar participants at the University of Oxford and the Technical University of Denmark. 


\section{References}

Best, M. J. and Grauer, R. R. (1991). On the Sensitivity of Mean-Variance-Efficient Portfolios to Changes in Asset Means: Some Analytical and Computational Results. Review of Financial Studies, 4(2):315-342.

Black, F. and Litterman, R. (1992). Global Portfolio Optimization. Financial Analysts Journal, 48(5):28-43.

Carhart, M. M. (1997). On Persistence in Mutual Fund Performance. The Journal of Finance, 52(1):57-82.

Chopra, V. K. and Ziemba, W. T. (1993). The Effect of Errors in Means, Variances, and Covariances on Optimal Portfolio Choice. The Journal of Portfolio Management, 19(2):6-11.

Clarke, R. G., de Silva, H., and Thorley, S. (2006). Minimum-Variance Portfolios in the U.S. Equity Market. The Journal of Portfolio Management, 33(1):10-24.

DeMiguel, V., Garlappi, L., Nogales, F. J., and Uppal, R. (2009a). A Generalized Approach to Portfolio Optimization: Improving Performance by Constraining Portfolio Norms. Management Science, 55(5):798-812.

DeMiguel, V., Garlappi, L., and Uppal, R. (2009b). Optimal Versus Naive Diversification: How Inefficient is the 1/N Portfolio Strategy? Review of Financial Studies, 22(5):1915-1953.

DeMiguel, V. and Nogales, F. J. (2009). Portfolio Selection with Robust Estimation. Operations Research, 57(3):560-577.

Duchin, R. and Levy, H. (2009). Markowitz Versus the Talmudic Portfolio Diversification Strategies. The Journal of Portfolio Management, 35(2):71-74.

Fama, E. F. and French, K. R. (1993). Common Risk Factors in the Returns on Stocks and Bonds. Journal of Financial Economics, 33(1):3-56.

Frazzini, A. and Pedersen, L. H. (2014). Betting Against Beta. Journal of Financial Economics, 111(1):1-25.

Hartigan, J. A. (1981). Consistency of Single Linkage for High-Density Clusters. Journal of the American Statistical Association, 76(374):388-394. 
Haugen, R. A. and Baker, N. L. (1991). The Efficient Market Inefficiency of Capitalization-weighted Stock Portfolios. The Journal of Portfolio Management, 17(3):35-40.

Jagannathan, R. and Ma, T. (2003). Risk Reduction in Large Portfolios: Why Imposing the Wrong Constraints Helps. The Journal of Finance, 58(4):1651-1684.

Jorion, P. (1986). Bayes-Stein Estimation for Portfolio Analysis. The Journal of Financial and Quantitative Analysis, 21(3):279-292.

Kan, R. and Zhou, G. (2007). Optimal Portfolio Choice with Parameter Uncertainty. Journal of Financial and Quantitative Analysis, 42(03):621-656.

Kritzman, M. (1993). What Practitioners Need to Know... About Factor Methods. Financial Analysts Journal, 49(1):12-15.

Kritzman, M., Page, S., and Turkington, D. (2010). In Defense of Optimization: The Fallacy of 1/ N. Financial Analysts Journal, 66(2):31-39.

Ledoit, O. and Wolf, M. (2003). Improved Estimation of the Covariance Matrix of Stock Returns with an Application to Portfolio Selection. Journal of Empirical Finance, 10(5):603-621.

Ledoit, O. and Wolf, M. (2004a). A Well-conditioned Estimator for Large-dimensional Covariance Matrices. Journal of Multivariate Analysis, 88(2):365-411.

Ledoit, O. and Wolf, M. (2004b). Honey, I Shrunk the Sample Covariance Matrix. The Journal of Portfolio Management, 30(4):110-119.

Ledoit, O. and Wolf, M. (2012). Nonlinear Shrinkage Estimation of Large-dimensional Covariance Matrices. The Annals of Statistics, 40(2):1024-1060.

Levy, H. and Levy, M. (2014). The Benefits of Differential Variance-based Constraints in Portfolio Optimization. European Journal of Operational Research, 234(2):372-381.

Lisi, F. and Corazza, M. (2008). Clustering Financial Data for Mutual Fund Management. In Perna, C. and Sibillo, M., editors, Mathematical and Statistical Methods in Insurance and Finance, pages 157-164. Springer Milan.

Markowitz, H. (1952). Portfolio Selection. The Journal of Finance, 7(1):77-91.

Merton, R. (1980). On Estimating the Expected Return on the Market: An Exploratory Investigation. Journal of Financial Economics, 8:323-361. 
Nanda, S., Mahanty, B., and Tiwari, M. (2010). Clustering Indian Stock Market Data for Portfolio Mnagement. Expert Systems with Applications, 37(12):8793-8798.

Scherer, B. (2011). A Note on the Returns from Minimum Variance Investing. Journal of Empirical Finance, 18(4):652-660.

Tan, P.-N., Steinbach, M., and Kumar, V. (2006). Introduction to Data Mining. Pearson.

Tobin, J. (1958). Liquidity Preference as Behavior Towards Risk. The Review of Economic Studies, 25(2):65-82.

Tola, V., Lillo, F., Gallegati, M., and Mantegna, R. N. (2008). Cluster Analysis for Portfolio Optimization. Journal of Economic Dynamics and Control, 32(1):235-258.

Tu, J. and Zhou, G. (2011). Markowitz meets Talmud: A Combination of Sophisticated and Naive Diversification Strategies. Journal of Financial Economics, 99(1):204-215.

$\mathrm{Xu}, \mathrm{R}$. and Wunsch, D. (2005). Survey of Clustering Algorithms. IEEE Transactions on Neural Networks, 16(3):645-78. 